\title{
Application of Computer Vision Technique on Sorting and Grading of Fruits and Vegetables
}

\section{Mahendran R*, Jayashree GC, Alagusundaram K}

Assistant professor, Indian Institute of Crop Processing Technology, Ministry of Food Processing Industries, GOI Pudukkottai Road, Thanjavur 613 005, Tamil Nadu, India

\begin{abstract}
The paper presents the recent development and application of image analysis and computer vision system in quality evaluation of products in the field of agriculture. Computer vision is a rapid, consistent and objective inspection technique, which has expanded into many diverse industries. Its speed and accuracy satisfy everincreasing production and quality requirements, hence aiding in the development of totally automated processes. The requirements and recent developments of hardware and software for machine vision systems are discussed, with emphases on monochrome imaging, colour imaging and multispectral imaging for modern grading and sorting systems. Examples of applications for detection of disease, defects, and contamination on fruits and vegetables are also given. Future trends of machine vision technology applications are discussed.
\end{abstract}

Keywords: Computer vision system; Image processing; Sorting; Grading

\section{Introduction}

Coding of pictures for transmission through cables was attempted way back in 1920 (even before the advent of digital computer), but serious attempts for digital image processing commenced in late $60 \mathrm{~s}$ and early 70s. Importance of computer vision increases due to the fact that vision is one of the most dominant human senses. The use of computer vision for the inspection of fruits and vegetables has increased during recent years. Computer vision technique has been a subject of research and application for more than four decades. This technique is used in many engineering fields such as robotics, industrial image processing, food processing and other fields. Quickness, nondestructive evaluation possibilities, easy procedures for application, quantum of output per unit time are some advantages favouring application of computer vision to engineering problems. Application of computer vision to food processing fields evolved first in 1989 for grain quality inspection [1].

Huge post harvest losses in handling and processing and the increased demand for food products of high quality and safety necessitates the growth of accurate, fast and objective quality determination of food and agricultural products [2]. Major areas of application of computer vision technology in food industry include quality evaluation of food grains, fruits, vegetables and processed foods such as chips, cheese and pizza. The technique had also found useful for determination insect infestation in grains and blemishes in fruits and vegetables.

The method used by the farmers and distributors to sort and grade agricultural and food products are through traditional quality inspection and handpicking which is time-consuming, laborious and less efficient. Manual sorting and grading are based on traditional visual quality inspection performed by human operators, which is tedious, time-consuming, slow and non-consistent. Harvesting traditionally is done by manual sensory observations. The quality attributes often used for deciding on the harvest maturity are color, appearance, texture and odor [3]. A first and an important step in the post harvest chain is sorting and grading of harvested produce. Commercially human senses are employed to sort or grade. Francis [4] found that human perception could easily be fooled. It is pertinent to explore the possibilities of adopting faster systems, which will save time and more accurate in sorting and grading of agricultural and food products. One of such reliable method is the automated computer vision system for sorting and grading. This paper reviews the progress of computer vision in the agricultural and food processing. A cost effective, consistent, superior speed and accurate sorting can be achieved using computer vision technique. The processing and manufacturing sectors requires automated inspection, as well as grading systems so that the losses incurred during harvesting, production and marketing can be minimized. Assisted sorting and grading of agricultural and food products is accomplished based on appearance, texture, colour, shape and sizes.

\section{Computer Vision System}

Computer Vision (CV) is the process of applying a range of technologies and methods to provide imaging-based automatic inspection, process control and robot guidance in industrial applications. While the scope of $\mathrm{CV}$ is broad and a comprehensive definition is difficult to distil, a generally accepted definition of computer vision is 'the analysis of images to extract data for controlling a process or activity' [5]. Computer vision is a novel technology for acquiring and analyzing an image of a real scene by computers to control machines or to process it. It includes capturing, processing and analyzing images to facilitate the objective and non-destructive assessment of visual quality characteristics in agricultural and food products [6]. The techniques used in image analysis include image acquisition, image pre-processing and image interpretation, leading to quantification and classification of images and objects of interest within images. Images are acquired with a physical image sensor and

${ }^{*}$ Corresponding author: Mahendran R, Indian Assistant professor, Indian Institute of Crop Processing Technology, Ministry of Food Processing Industries, GOI Pudukkottai Road, Thanjavur 613 005, Tamil Nadu, India, Tel: 04362 228155/226676; Fax: 04362-227971; E-mail: mahendran@iicpt.edu.in

Received June 23, 2012; Accepted August 02, 2012; Published August 07, 2012

Citation: Mahendran R, Jayashree GC, Alagusundaram K (2012) Application of Computer Vision Technique on Sorting and Grading of Fruits and Vegetables. J Food Process Technol S1-001. doi:10.4172/2157-7110.S1-001

Copyright: (c) 2012 Mahendran R, et al. This is an open-access article distributed under the terms of the Creative Commons Attribution License, which permits unrestricted use, distribution, and reproduction in any medium, provided the original author and source are credited. 
dedicated computing hardware and software are used to analyze the images with the objective of performing a predefined visual task.

\section{Size, shape and color analysis}

Size, which is the first parameter identified with quality, has been estimated using machine vision by measuring either projected area $[7,8]$, perimeter [9] or diameter [10]. Size measurement is important for determining produce surface area. The shape is one of the important visual quality parameters of fruits, vegetables, etc. Currently human sorters are employed to sort fruits based on shape. Shape is a feature, easily comprehended by human but difficult to quantify or define by computer. Most of the machine vision shape detection work has been done on industrial objects, which have definite structure. Agricultural and biological products are unique in nature and the growing environment causes various boundary irregularities which influences their shapes. Image processing offers solution for sorting of fruits based on their shape. Colour is also an important quality factor that has been widely studied [11-14]. The colour of an object is determined by wavelength of light reflected from its surface. In biological materials the light varies widely as a function of wavelength. These spectral variations provide a unique key to machine vision and image analysis.

Some fruits have one colour homogeneously distributed on the skin surface, which is called primary colour. The averaged surface colour is a good quality indicator for these fruits. However, other fruits (e.g. some varieties of peaches, apples, tomatoes) have a secondary colour that can be used as a good indicator of maturity. In this case, it is not possible to rely only on the global colour as a quality parameter.

Majumdar and Jayas [15], developed a digital image analysis (DIA) algorithm based on color features to classify individual kernels of Canada Western Red Spring (CWRS) wheat, Canada Western Amber Durum (CWAD) wheat, barley, oats, and rye. Eighteen color features (mean, variance, range of red, green, blue, hue, saturation, and intensity) were used for the discriminant analysis. Grains from 15 growing regions (300 kernels per growing region) were used as the training data set and another five growing regions were used as the test data set. When the first 10 most significant color features were used in the color model and tested on an independent data set (the test data set where total number of kernels used was 10,500; for CWRS wheat, 300 kernels each were selected for three grades), the classification accuracies of CWRS wheat, CWAD wheat, barley, oats, and rye were 94.1, 92.3, 93.1, 95.2, and $92.5 \%$ respectively. When the model was tested on the training data set (total number of kernels used was 31,500), the classification accuracies were 95.7, 94.4, 94.2, 97.6, and 92.5\%, respectively, for CWRS wheat, CWAD wheat, barley, oats, and rye.

Blasco et al. [16] developed computer vision techniques for online estimation of the quality of oranges, peaches and apples based on quality attributes: size, colour, stem location and detection of external blemishes. The segmentation procedure, based on a Bayesian discriminant analysis, allowed fruits to be precisely distinguished from the background. To evaluate the accuracy of colour estimation by the developed sensor, colour measurements were taken from 22 surface sectors of several tomatoes. These sectors varied from a red-greenish colour to red. The ability of the machine vision system to determine fruit colour was evaluated by comparing several standard colour indices that are commonly used for different fruits. These indices were calculated from Hunter Lab co-ordinate values provided by a colorimeter in three random circular areas ( $8 \mathrm{~mm}$ diameter) in each of the selected sectors.

\section{Hardware}

The hardware configuration of computer-based Computer vision systems is relatively standard [2]. Typically, a computer vision system consists of:

- An illumination device, which illuminates the sample under test

- A solid-state Charged Coupled Device array camera, to acquire an image

- A frame-grabber, to perform the A/D (analog-to-digital) conversion of scan lines into picture elements or pixels digitized in a $\mathrm{N}$ row by $\mathrm{M}$ column image

- A personal computer or microprocessor system, to provide disk storage of images and computational capability with software and specific application programs

- A high-resolution colour monitor, which aids in visualizing the images and the effects of various image analysis routines, on the images.

\section{Software}

The image analysis is performed by a specific software application developed at IVIA (Instituto Valenciano de Investigaciones Agrarias) using the programming language $\mathrm{C}, \mathrm{C}++, \mathrm{MATLAB}$. The software was divided into two modules: an application for training the system; and another to command the acquisition, process the images and provide the estimated quality parameters of each fruit [16].

\section{Image capturing method}

Pixel: A pixel is a single point picture element in a graphic image. A digital image, however, is discrete in nature and there is some point, which cannot be magnified further. This point is called a pixel of that digital image. Graphics monitors display pictures by dividing the display screen into thousands (or millions) of pixels, arranged in rows and columns. The pixels are so close together that they appear connected. The number of bits used to represent each pixel determines how many colors or shades of gray can be displayed. For example, in 8 -bit color mode, the color monitor uses 8 bits for each pixel, making it possible to display 2 to the 8 th power (256) different colors or shades of gray. On color monitors, each pixel is actually composed of three dots a red, a blue, and a green dot. Ideally, the three dots should all converge at the same point, but all monitors have some convergence error that can make color pixels appear fuzzy. The quality of a display system largely depends on its resolution, how many pixels it can display, and how many bits are used to represent each pixel.

\section{Image acquisition}

A digital image is a numeric representation (normally binary) of a two dimensional image. Depending on whether or not the image resolution is fixed, it may be of vector or raster type. Digital images are electronic snapshots taken of a scene or scanned from documents such as photographs, manuscripts, printed texts and art work. Image acquisition is the transfer of the electronic signal from the sensing device into a numeric form. Image pre-processing refers to the initial processing of the raw image data for correction of geometric distortions, removal of noise, grey level correction and correction for blurring [17].

Singh and Delwiche employed a single CCD video camera mounted above the sample to capture image details for detecting and identifying 
major peach defects. Three images were taken of each peach to cover the entire surface, thus showing a need to acquire detailed image features. Shape was quantified in two dimensional spaces by the eccentricity of the peach, as the maximum dimension divided by the minimum dimension of the peach. The technology aims to duplicate the effect of human vision by electronically perceiving and understanding an image [18].

CCDs are sensors used in digital cameras and video cameras to record still and moving images. The CCD captures light and converts it to digital data that is recorded by the camera. For this reason, a CCD is often considered the digital version of film and is a major technology for digital imaging. In a CCD image sensor, reverse-biased $\mathrm{p}-\mathrm{n}$ junctions (essentially photodiodes) are used to absorb photons and produce charges representing sensed pixels; the CCD is used to read out these charges. Although CCDs are not the only technology to allow for light detection, CCD image sensors are widely used in professional, medical, and scientific applications where high-quality image data is required. Typically the image sensors used in machine vision are usually based on solid state charged coupled device (CCD) camera technology with some applications using thermionic tube (vacuum tube) devices. CCD cameras are either of the array type or line scan type. Array or area type cameras consist of a matrix of minute photosensitive elements (photosites) from which the complete image of the object is obtained based on output proportional to the amount of incident light. Alternatively, line scan cameras use a single line of photosites which are repeatedly scanned up to 2000 times per minute to provide an accurate image of the object as it moves under the sensor [19].

Images were captured using an image acquisition system for color digital camera similar to that developed by Papadakis et al. [20] (Figure 1). Samples were illuminated using four fluorescent lamps (length of $60 \mathrm{~cm}$ ) with a color temperature of $650^{\circ} \mathrm{C}$ (Philips, Natural Daylight, $18 \mathrm{~W}$ ) and a color rendering index (Ra) close to $95 \%$. The four lamps were arranged as a square $35 \mathrm{~cm}$ above the sample and at an angle of 45 with the sample plane to give a uniform light intensity over the food sample. A color digital camera (CDC) Power Shot G3 (Canon, Japan) was located vertically at a distance of $22.5 \mathrm{~cm}$ from the sample. The angle between the camera lens axis and the lighting sources was around 45. Sample illuminators and the CDC were inside a wood box whose internal walls were painted black to avoid the light and reflection from the room. The white balance of the camera was set using a standardized gray color chart from Kodak. Color standards were photographed and analyzed periodically to ensure that the lighting system and the CDC were working properly. Images were captured with the mentioned CDC at its maximum resolution (2272.1704 pixels) and connected to the USB port of a Pentium IV, $1200 \mathrm{MHz}$ computer. Canon Remote Capture Software (version 2.7.0) was used for acquiring the images directly in the computer in TIFF format without compression.

\section{Monochrome imaging}

Monochrome imaging requires a single-chip CCD. The resolution of a CCD image depends on how many pixels are in the CCD arrays. Depending on the nature of applications, the camera resolution can range from 480 to 1024 lines or even higher. It is used for the detection of blemishes and bruises on apples [21-23]. Monochrome machine vision technology was also used for detecting scars, cracks, and spreading tips for asparagus [24]. Grading apples with on-line machine vision has been attempted $[22,25]$. The major challenges for on-line inspection are to produce quality images that provide clearly identifiable features

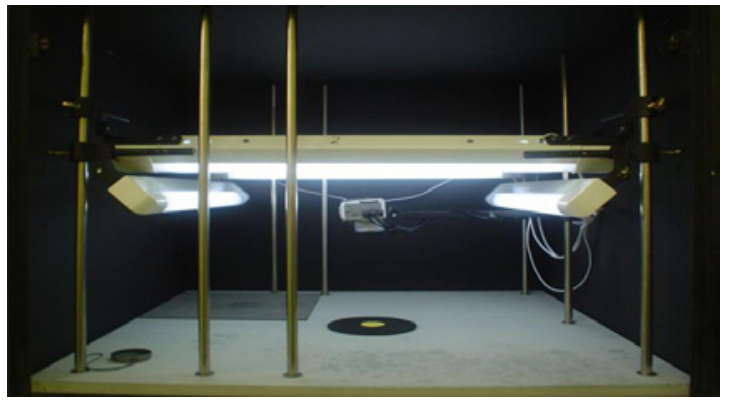

Figure 1: Potato chip image acquisition system.

and to have both efficient hardware and software to process the images fast enough for on-line implementation.

\section{Color imaging}

Color features of fruits and vegetables included mean, variance, ranges of the red (R), green $(G)$, and blue (B) color primaries and the derived hue $(\mathrm{H})$, saturation $(\mathrm{S})$, and intensity (I) values. The 256 grey levels of the R, G, and B values were grouped in bands of 16 and were called the histogram features. A single chip CCD camera can also be used for color imaging. This is done by alternating the pixels in the CCD camera for red, green and blue (RGB) color acquisition in the area array $\mathrm{CCD}$ to simulate the colours seen by the human eye. Color imaging can also be achieved using three-chip CCD camera systems. Each CCD in a three-chip camera receives RGB colours to produce nearly true color images of the objects. This is accomplished using a prism assembly with band pass filters and a dichroic coating on selected surfaces of the prisms that separate broad band light into RGB channels. Throop et al. [26] used a color difference between bruised and non-bruised regions on 'Golden Delicious' apples. Daley et al. [27,28] applied color imaging techniques to on-line poultry quality grading.

\section{Illumination}

Illumination is an important prerequisite of image acquisition for food quality evaluation. The quality of captured image can be greatly affected by the lighting condition. A high quality image can help to reduce the time and complexity of the subsequent image processing steps, which can decrease the cost of an image processing system. Different applications may require different illumination strategies. The performance of the illumination system greatly influences the quality of image and plays an important role in the overall efficiency and accuracy of the system. Lighting type, location and colour quality play important roles in bringing out a clear image of the object. Lighting arrangements are grouped into front- or back-lighting. Front lighting serve as illumination focusing on the object for detection of external surface features of the product while back-lighting is used for enhancing the background of the object.

Light sources also differ but may include incandescent, fluorescent, lasers, X-ray tubes and infrared lamps. The choice of lamp affects quality and image analysis performance [29]. Sarkar [30] found that by adjustment of the lighting, the appearance of an object can be radically changed with the feature of interest clarified or blurred (Figure 2, Arivazhagan et al.) [31]. When radiation from the lighting system illuminates an object, it is transmitted through, reflected, or absorbed. These phenomena are referred to as optical properties. The absorbed light can also be re-emitted (fluorescence), usually at longer wavelengths. A number of compounds emit fluorescence in the visible 
region of the spectrum when excited with UV radiation. The optical properties and fluorescence emission from the object are integrated functions of the angle and wavelength of the incident light and chemical and physical composition of the object.

\section{Image processing}

Image processing in agricultural applications consist of three steps: (1) image enhancement, (2) image feature extraction and (3) image feature classification. Image enhancement is commonly applied to a digital image to correct problems such as poor contrast or noise. Image enhancement procedures such as morphological operations, filters, and pixel-to-pixel operations are generally used to correct inconsistencies in the acquired images caused by inadequate and/or non-uniform illumination. Statistical procedures from basic image statistics such as mean, standard deviation, and variance to more complex measurement such as principle component analysis can be used to extract features from digital images.

For the test fruit image, color and texture features are derived as that of the training phase and compared with corresponding feature values, stored in the feature library. The classification is done using the Minimum Distance Criterion. The image from the training set which has the minimum distance when compared with the test image says that the test image belongs to the category of that training image. (Figure 3, Arivazhagan et al.) [31]

Once image features are identified, the next step is feature classification. Numerical techniques such as neural networks and fuzzy inference systems have been successfully applied to perform image feature classification. Pre-processing refers to the initial processing of the raw image. The images captured or taken are transferred onto a computer and are converted to digital images. Digital images though displayed on the screen as pictures, are digits, which are readable by the computer and are converted to tiny dots or picture elements representing the real objects. In some cases pre-processing is done to improve the image quality by suppressing undesired distortions referred to as "noise" or by the enhancement of important features of interest. The intermediate-level processing involves image segmentation, image representation and image description.

\section{Image segmentation}

Image segmentation is a process of cutting, adding and feature
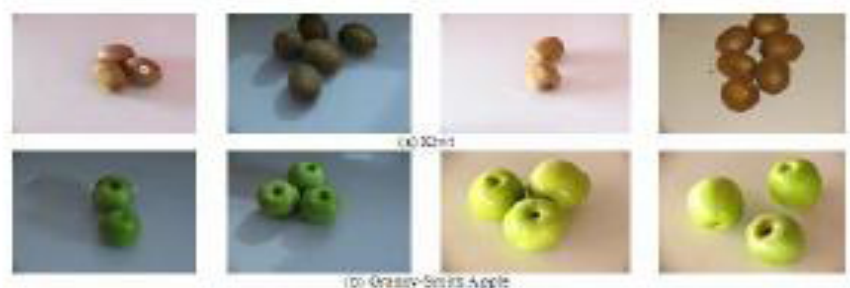

Figure 2: Illumination Difference.

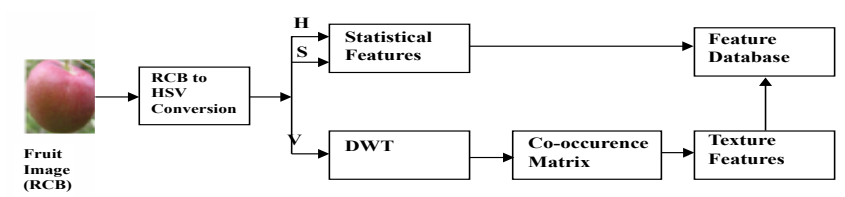

Figure 3: Feature Extraction. analysis of images aimed at dividing an image into regions that have a strong correlation with objects or areas of interest using the principle of matrix analysis. Image segmentation is one of the most important steps in the entire image processing technique, as subsequent extracted data are highly dependent on the accuracy of this operation. Its main aim is to divide an image into regions that have a strong correlation with objects or areas of interest. If objects in image cannot be segmented correctly, it is difficult for object measurement; classification and recognition, hence impact interpreting and understanding that image. Earlier studies proposed the use of a 'flooding' algorithm to segment patch-like defects (russet patch, bruise, and also stalk or calyx area) [32]. It was found that this method of feature identification is applicable to other types of produce with uniform skin colour. This technique was improved by Yang and Marchant [33], who applied a 'snake' algorithm to closely surround the defects. Segmentation can be achieved by three different techniques: thresholding, edge-based segmentation and region-based segmentation as shown in Figure 4: (Sonka et al.; Sun) $[18,34]$. Thresholding is a simple and fast technique for characterising image regions based on constant reflectivity or light absorption of their surfaces. Edge-based segmentation relies on edge detection by edge operators. Edge operators detect discontinuities in grey level, colour, texture. Region segmentation involves the grouping together of similar pixels to form regions representing single objects within the image. Raji et al. [35] developed a programme in FORTRAN using the principle of edge detection in image analysis to determine the edge of sliced breads and biscuits (round and rectangular) with a view to detect defects (breakage).

Computer vision has been used for such tasks as shape classification, defect detection, quality grading and variety classification. A colour model developed was used as a standard for comparison with sample images. The developed algorithm gave satisfactory results with wellcontrasted defects; however two further enhancements following segmentation were required to improve accuracy. A novel adaptive spherical transform was developed and applied in a machine vision defect sorting system [36]. The transform converts a spherical object image to a planar object image allowing fast feature extraction, giving the system an inspection capacity of 3000 apples $\mathrm{min}^{-1}$ from the three cameras, each covering 24 apples in the field of view. A $94 \%$ success rate was achieved for sorting defective apples from good ones for the 600 samples tested [37].

\section{Image analysis}

Image analysis is the process of distinguishing the objects (regions of interest) from the background and producing quantitative information, which is used in the subsequent control systems for decision making. Luis Gracia et al. [38] present the flowchart of the developed application for image analysis as shown in the Figure 5. The first step is image acquisition, which includes the software filtering described in subsection "Erode and Dilate process". Next, the image is binarized. After that, the opening/closing operations and the erode/ dilate algorithms are applied, hence only relevant blobs (i.e., the flowers/ fruits/vegetables) remain in the image. The "feature extraction" step obtains the characterization of each detected blobs, i.e. the position of the centre of the flower/fruit/vegetable and the cutting point that allows separating the element from its peduncle. Finally, the "movement action" step sends the characterization information of each flower/ fruit/vegetable to the robotic mechanism responsible for the desired task: recollection, cutting, packaging, classification, fumigation, etc. 


\section{Color analysis based on histogram}

In image processing and photography, a color histogram is a representation of the distribution of colors in an image. For digital images, a color histogram represents the number of pixels that have colors in each of a fixed list of color ranges that span the image's color space, the set of all possible colors. The color histogram can be built for any kind of color space, although the term is more often used for threedimensional spaces like RGB or HSV. For monochromatic images, the term intensity histogram may be used instead. For multi-spectral images, where each pixel is represented by an arbitrary number of measurements (for example, beyond the three measurements in RGB), the color histogram is $\mathrm{N}$-dimensional, with $\mathrm{N}$ being the number of measurements taken. Each measurement has its own wavelength range of the light spectrum, some of which may be outside the visible spectrum. In case of colour image, in the RGB colour space, every individual colour component, namely red, green and blue has its histogram. Then, the percentage composition of every individual colour component, which a fruit possesses are to be evaluated. Using this percentage composition the level for a component can be set as a standard in classifying the apples based on a particular colour orientation. For an Apple the higher percentage composition of the red component was assigned the superior grade, the next lower composition the second grade and likewise the descending grades were assigned. This enabled the sorting of apples based on the colour as a parameter.

\section{Image interpretation}

The methodology was developed previously and is carefully detailed by Leon et al. [39]. Five models for the conversion from RGB images to $L^{\star} a^{\star} b^{\star}$ units were developed and tested: linear, quadratic, gamma, direct, and neural network. In the evaluation of the performance of those models, the neural network model stands out with an error of only $0.96 \%$. So it was possible to find in each pixel of the image $\mathrm{L}^{*} \mathrm{a}^{*} \mathrm{~b}^{*}$

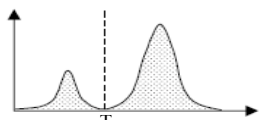

(a)

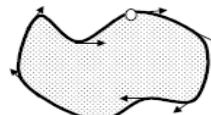

(b)

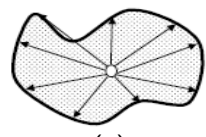

(c)
Figure 4: Typical segmentation techniques (a) Thresholding, (b) edge-based segmentation and (c) region based segmentation (Sun, 2000).

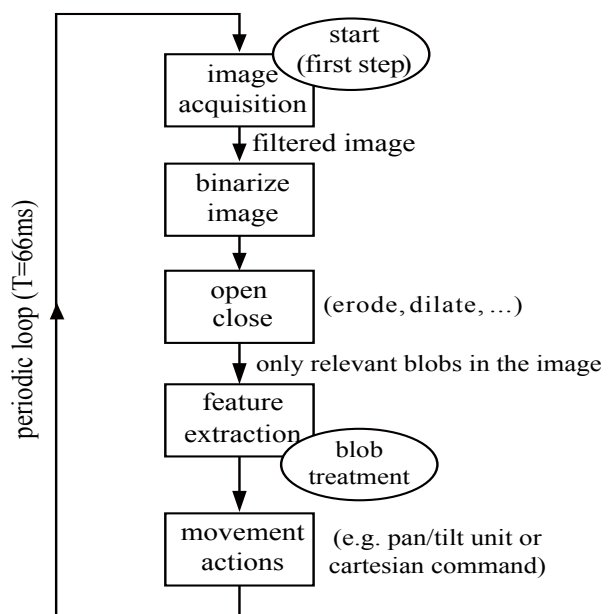

Figure 5: Flowchart of the of the developed application. color measuring system that is appropriate for an accurate, exact and detailed characterization of a food item, thus improving quality control and providing a highly useful tool for the food industry based on a color digital camera.

\section{Sorting}

Automatic fruit sorting can improve the quality of the product, abolish inconsistent manual evaluation, and reduce dependence on available manpower. Quality sorting is based on a multitude of measures, flavor (sweetness, acidity), appearance (color, size, shape, blemishes, glossiness), and texture (firmness, mouthfeel). Edan [40] indicated that multi-sensor quality classification can be applied in real sorting and improves the overall classification. Quality classification of tomatoes was successfully applied using vision and an impact sensor [41]. To sort according to the surface defects requires an analysis as complete as possible of the entire fruit surface. The company working with the CEMAGREF Institute in France, in this research has produced for its automatic colour sorting system (already marketed) a satisfactory conveyor belt. This conveyor belt, built with rotary rubber rollers, carries the fruit along while they rotate. This rotation enables the colour detection system or a human observer to look at most of the surface of the fruit. But, with this system, it is not possible to inspect the poles. The shape of the rubber rollers (bicones) enables the fruit to be aligned in three to eight lines across the conveyor belt as shown in Figure 6 and 7. The company had asked CEMAGREF to integrate the new processing system for blemish detection into it. The estimation of the surface defects, unlike the colour sorting, requires a very precise analysis of the fruit. Even a small damaged area can downgrade the fruit. The system must be able to analyse elementary surfaces of a few square millimetres. To do this, cameras were used as sensors. The light colour of the Golden Delicious apples allows examination by a monochromatic camera with a suitable filter.

Delwiche et al. [11] developed a multi-camera, multi-processor system to sort prunes for surface defects in real time. Three line-scan cameras were used to view the prune, thus showing again the need for acquiring three-dimensional images. With a combined sample of prunes containing $28 \%$ defective fruit, $6 \%$ for good prunes and $8 \%$ for defective prunes that utilized an image acquisition system with two windows in the sides of the vision chamber allowing charge coupled device (CCD) video camera mounting. The relationship between object shape and its boundary spectrum values in Fourier domain was explored for shape extraction. A shape separator based on harmonics of the Fourier transform was designed in a two-dimensional space for potato shape separation.

\section{Conclusion}

The paper presents the recent developments in computer vision system in the field of agricultural and food products. The adoption of this emerging technology in sorting and grading of fruits and vegetables will be of immense benefit to this country. Some of the other associated benefits include more efficient operation, production of more consistent product quality, greater product stability and safety. Computer vision systems have been used increasingly in industry for inspection and evaluation purposes as they can provide rapid, economic, hygienic, consistent and objective assessment. However, difficulties still exist, evident from the relatively slow commercial uptake of computer vision technology in all sectors. Even though adequately efficient and accurate algorithms have been produced, processing speeds still fail to meet modern manufacturing requirements. With few exceptions, research 
Citation: Mahendran R, Jayashree GC, Alagusundaram K (2012) Application of Computer Vision Technique on Sorting and Grading of Fruits and Vegetables. J Food Process Technol S1-001. doi:10.4172/2157-7110.S1-001

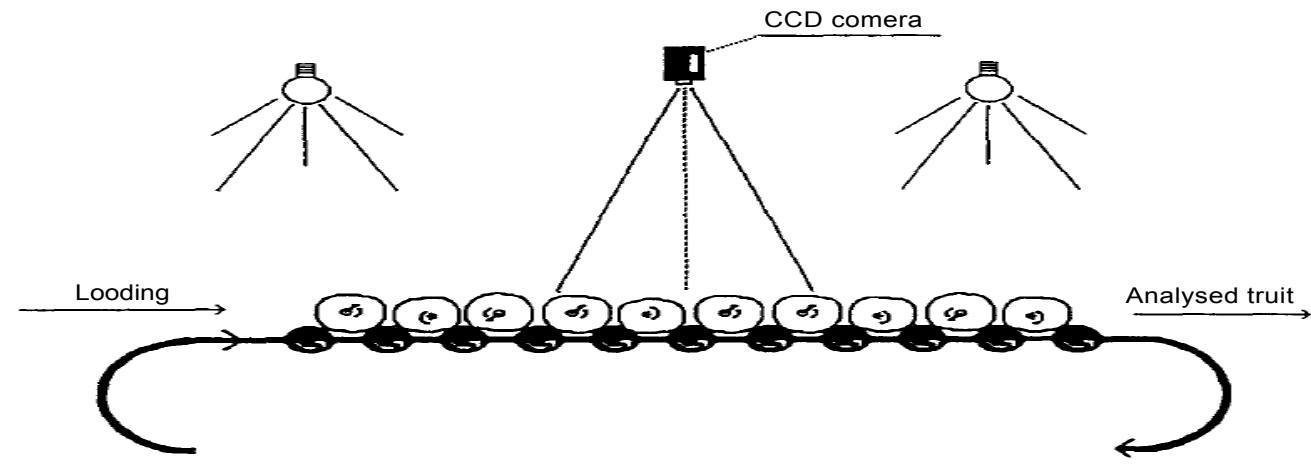

Figure 6: Lateral view of the conveyor belt.

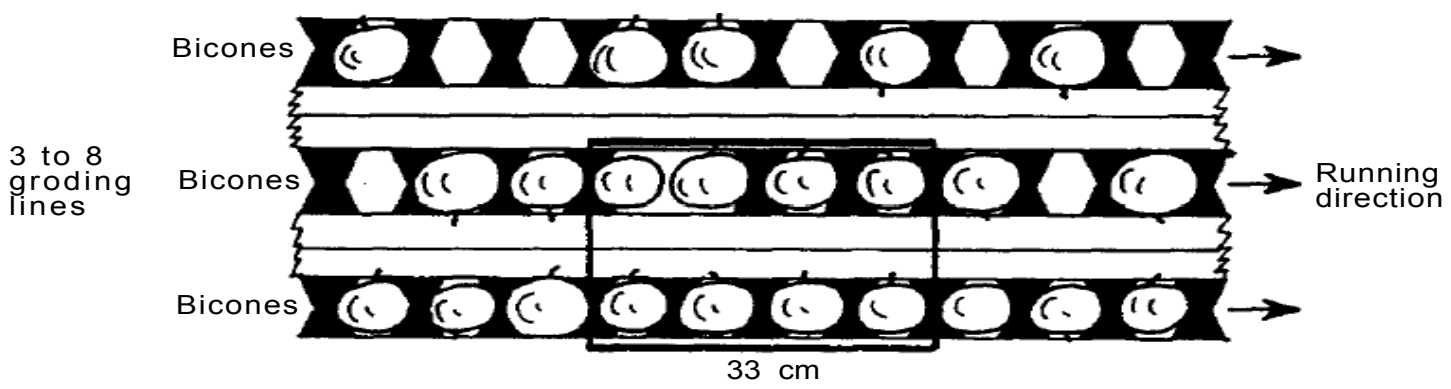

Figure 7: Plan view of the conveyor.

in this field has dealt with trials on a laboratory scales and hence it needs more focused and detailed study.

\section{References}

1. Zayas I, Pomeranz Y, Lai FS (1989) Discrimination of wheat and non wheat components in grain samples by image analysis. Cereal Chemistry 66: 233237

2. Narendra VG, Hareesh KS (2010) Prospects of computer vision automated grading and sorting systems in agricultural and food products for quality evaluation. Int J Comp App 1: $0975-8887$.

3. Sun DW, Brosnan T (2003) Improving quality inspection of food products by computer vision: a review. J Food Engineering 61: 3-16.

4. Francis FJ (1980) Colour quality evaluation of horticultural crops. HortScience 15: $14-15$.

5. Relf Christopher G (2004) Image Acquisition and Processing with LabVIEW, Taylor \& Francis, pp. 1-264

6. Timmermans AJM (1998) Computer vision system for online sorting of pot plants using an artificial neural network classifier. Acta Horticulturae 421: 91 98.

7. Tao Y, Morrow CT, Heinemann PH, Sommer JH (1990) Automated machine vision inspection of potatoes. ASAE 90-3531.

8. Varghese Z, Morrow CT, Heinemann PH, Sommer HJ, Tao Y, et al. (1991) Automated inspection of Golden Delicious apples using colour computer vision. ASAE 91-7002.

9. Sarkar N, Wolfe RR (1985) Feature extraction techniques for sorting tomatoes by computer vision. Transactions of the ASABE 28: 970-974.

10. Brodie JR, Hansen AC, Reid JF (1994) Size assessment of stacked logs via the hough transform. Transactions of the ASAE 37: 303-310.

11. Singh N, Delwiche MJ, Johnson RS, Thompson J (1992) Peach maturity grading with color computer vision. ASAE 92-3029.
12. Singh N, Delwiche MJ, Johnson RS (1993) Image analysis methods for realtime color grading of stonefruit. Computers and Electronics in Agriculture 9 : 71-84.

13. Hahn F (2002) Multispectral prediction of unripe tomatoes. Biosystems Engineering 81: 147-155.

14. Dobrzanski B, Rybczynski R (2002) Colour change of apple as a result of storage, shelf-life, and bruising. International Agrophysics 16: 261-268.

15. Majumdar S, Jayas DS (2000) Classification of cereal grains using machine vision: II Color models. Transactions of the ASAE 43: 1677-1680.

16. Blasco J, Aleixos N, Molto E (2003) Machine vision system for automatic quality grading of fruit. Biosystems Engineering 85: 415-423.

17. Shirai Y (1987) Three dimensional computer vision. Berlin: Springer-Verlag pp. 1-297.

18. Sonka M, Hlavac V, Boyle R (1999) Image processing, analysis, and machine vision. California, USA: PWS Publishing.

19. Wallin P, Haycock P (1998) Foreign body prevention, detection and control: A Practical Approach. 4: 359.

20. Papadakis SE, Abdul-Malek S, Kamdem RE, Yam KL (2000) A versatile and inexpensive technique for measuring color of foods. Food Technology 5: 48-51.

21. Davenel A, Guizard C, Labarre T, Sevila F (1988) Automatic detection of surface defects on fruit using a vision system. J Agric Eng Res 41: 1-9.

22. Rehkugler GE, Throop JA (1989) Image processing algorithm for apple defect detection. Trans ASABE 32: 267-272.

23. Singh N, Delwiche MJ (1994) Machine vision methods for defect sorting stone fruit. Transactions ASAE 37: 1989-1997.

24. Rigney MP, Brusewitz GH, Stone ML (1996) Peach physical characteristics for orientation. Trans ASAE 39: 1493-1497.

25. Throop JA, Aneshansley DJ, Upchurch BL (1995) An image processing algorithm to find new and old bruises. Appl Eng Agric 11: 751-757. 
Citation: Mahendran R, Jayashree GC, Alagusundaram K (2012) Application of Computer Vision Technique on Sorting and Grading of Fruits and Vegetables. J Food Process Technol S1-001. doi:10.4172/2157-7110.S1-001

26. Throop JA, Aneshansley DJ, Upchurch BL (1993) Near-IR and color imaging for bruise detection on Golden Delicious apples. Proc SPIE 1836: 33-44.

27. Daley W, Carey R, Thompson C (1993) Poultry grading/inspection using color imaging. Proc SPIE 1907: 124-132.

28. Daley W, Carey R, Thompson C (1995) Real-time color grading and defect detection of food products. Proc SPIE 2345: 403-411.

29. Bachelor BG (1985) Lighting and viewing techniques in automated visual inspection. Bedford, UK: IFSPublication Ltd. Computers and Electronics in Agriculture 20: 117-130.

30. Sarkar NR (1991) Machine vision for quality control in the food industry. In I Fung \& E Matthews (Eds.), Instrumental methods for quality assurance in foods, pp. 167-187. New York: Marcel Decker.

31. Arivazhagan.S, Newlin Shebiah R, Selva Nidhyanandhan S, Ganesan L (2010) Fruit recognition using color and texture features. Journal of Emerging Trends in Computing and Information Sciences 1: 90-94.

32. Yang Q (1994) An approach to apple surface feature detection by machine vision. Computers and Electronics in Agriculture 11: 249-264.

33. Yang Q, Marchant JA (1995) Accurate blemish detection with active contour models. Computers and Electronics in Agriculture 14: 77-89.

34. Sun DW (2000). Inspecting pizza topping percentage and distribution by a computer vision method. Journal of Food Engineering 44: 245-249.
35. Raji AO, Fagboun AA, Dania MK (2000) An approach to detecting defects in food products, Proceedings of the First International Conference of the Nigerian Institution of Agricultural Engineers 36-39.

36. Tao Y, Wen Z (1999) An adaptive spherical image transform for high-speed fruit defect detection. Transaction of the ASAE 42: 241-246.

37. Tadhg Brosnan, Da-Wen Sun (2002) Inspection and grading of agricultura and food products by computer vision systems-a review. Computers and Electronics in Agriculture 36: 193-213.

38. Luis Gracia, Carlos Perez-Vidal, Carlos Gracia (2011) Computer vision applied to flower, fruit and vegetable processing. World Academy of Science Engineering and Technology 78: 430-436.

39. Leon K, Mery D, Pedreschi F, Leon J (2006) Color measurements in L*a*b* units from RGB digtal images. Food Research International 39: 1084-1091.

40. Edan Y, Shmulevich I, Rachmani D, Fallik E, Grinberg S (1994) Neural networks for quality grading of Tomatoes based on mechanical properties. Proceedings of the Food Automation III Conference: 346-355.

41. Edan Y, Pasternak H, Shmulevich I, Rachmani D, Guedalia D, et al. (1997) Color and firmness classification of fresh market tomatoes. Journal of Food Science 62: 793-796.

This article was originally published in a special issue, Food Processing handled by Editor(s). Dr. Tony Jin, USDAâ $€^{\mathrm{TM}_{\mathrm{S}}}$ Eastern Regional Research Center, USA 